\title{
Special Issue on Micro-2014, 1st International Conference on Microelectronics, Circuits and Systems, Kolkata, India, July 2014
}

\author{
Jyotsna Kumar Mandal ${ }^{1} \cdot$ Dulal Acharjee $^{2} \cdot$ Bernd Michel $^{3}$
}

Published online: 15 October 2016

(C) Springer-Verlag Berlin Heidelberg 2016

In the extended version of the first International Conference on Microelectronics Circuits and Systems, held on 11-13th July 2014 at Science City, Kolkata, India. 68 accepted papers were presented during 2 days of the conference. Out of these papers, 23 papers were initially selected for extended version for the MST Journal, Springer with the criteria that the title of the paper should be changed, at least $30-40 \%$ content of the article should be new and innovative and there should be a comparison with state-of-the-art of existing articles. The authors were requested to send the articles to the guest editor of the MST first in offline mode for the scrutiny regarding incorporation of the criteria framed. Then they were permitted to upload the paper to the MST journal through the editorial manager portal. The arrangement for peer reviews was made. At least three domain experts across the globe reviewed the papers.
Ultimately 14 papers were selected for publication in the MST Journal.

On behalf of the Organization and Program Committee of the conference we would like to thank the authority of MST, Springer to provide us opportunity to work as editors of the special issue. Also thanks to all reviewers of the articles for their esteem effort to uphold the spirit of the special issue of the Springer Microsystem Technology Journal.

We hope the articles will be useful for the researchers and engineers.

Jyotsna Kumar Mandal

Conference Chair Micro-2014

Dulal Acharjee

General Chair Micro-2014

Bernd Michel

Editor-in-Chief Journal Microsystem Technologies

Jyotsna Kumar Mandal

jkm.cse@gmail.com

1 University of Kalyani, West Bengal, India

2 Applied Computer Technology, Kolkata, India

3 Micro Materials Center Berlin, Berlin, Germany 